\title{
Patient with multiple acyl-CoA dehydrogenase deficiency disease and ETFDH mutations benefits from riboflavin therapy: a case report
}

\author{
Liuh Ling Goh', Yingshan Lee ${ }^{2}$, Ee Shien Tan ${ }^{3}$, James Soon Chuan Lim ${ }^{4}$, Chia Wei Lim ${ }^{1}$ and Rinkoo Dalan²,5,6*
}

\begin{abstract}
Background: Lipid storage myopathy (LSM) is a diverse group of lipid metabolic disorders with great variations in the clinical phenotype and age of onset. Classical multiple acyl-CoA dehydrogenase deficiency (MADD) is known to occur secondary to mutations in electron transfer flavoprotein dehydrogenase (ETFDH) gene. Whole exome sequencing (WES) with clinical correlations can be useful in identifying genomic alterations for targeted therapy.

Case presentation: We report a patient presented with severe muscle weakness and exercise intolerance, suggestive of LSM. Diagnostic testing demonstrated lipid accumulation in muscle fibres and elevated plasma acyl carnitine levels. Exome sequencing of the proband and two of his unaffected siblings revealed compound heterozygous mutations, $c$. 250G > A (p.Ala84Thr) and c.770A > G (p.Tyr257Cys) in the ETFDH gene as the probable causative mutations. In addition, a previously unreported variant c.1042C > T (p.Arg348Trp) in ACOT11 gene was found. This missense variant was predicted to be deleterious but its association with lipid storage in muscle is unclear. The diagnosis of MADD was established and the patient was treated with riboflavin which resulted in rapid clinical and biochemical improvement.
\end{abstract}

Conclusions: Our findings support the role of WES as an effective tool in the diagnosis of highly heterogeneous disease and this has important implications in the therapeutic strategy of LSM treatment.

Keywords: ETFDH, Lipid storage myopathy, Multiple acyl-CoA dehydrogenase deficiency, Whole exome sequencing

\section{Background}

Lipid storage myopathy (LSM) is a diverse group of lipid metabolic disorders characterized by impaired fatty acids oxidation [1]. Multiple acyl-coenzyme A dehydrogenase deficiency (MADD) is an autosomal recessive disorder characterized by mitochondrial electron transfer system defects and impaired fatty acids metabolism [2]. MADD is associated with a highly diverse clinical phenotype, ranging from the lethal neonatal onset type with congenital anomalies, to the adult onset type with milder and variable clinical presentation [3]. The clinical heterogeneity of adult-onset forms pose a challenge for

\footnotetext{
* Correspondence: Rinkoo_Dalan@ttsh.com.sg

${ }^{2}$ Department of Endocrinology, Tan Tock Seng Hospital, 11 Jalan Tan Tock Seng, Singapore 308433, Singapore

${ }^{5}$ Lee Kong Chian School of Medicine, Nanyang Technological University, 11

Mandalay Road, Singapore 308232, Singapore

Full list of author information is available at the end of the article
}

diagnosis with the vast majority displaying mild or atypical biochemical abnormalities [4]. Diagnosis of MADD is aided by urinary organic acid analysis and blood acylcarnitine profile.

Classical MADD has been known to occur secondary to deficiency in electron transfer flavoprotein (ETF) or ETF:ubiquinone oxidoreductase (ETF:QO). The severity of the disease is dependent on the location and nature of mutations in the genes encoding ETF or ETF:QO. Null mutations result in a complete loss of protein expression or function leading to lethal disease. Missense mutations result in only a partial loss of enzyme activity, with preservation of some residual enzyme activity thus leading to a milder clinical phenotype [5-7].

Some MADD patients have an improvement in the clinical symptoms and metabolic profile with riboflavin treatment. These patients, referred to as riboflavin-responsive 
MADD (RR-MADD) patients, have been seen to harbour variations in the ETFDH gene encoding ETF:QO. To date, more than 50 different ETFDH variations have been reported in RR-MADD [8-12]. The ETF:QO protein consists of a flavin adenine dinucleotide (FAD) binding domain and a [4Fe-4S] cluster. This protein is responsible for ATP production by mediating the transfer of reducing equivalents to the respiratory ubiquinone pool $[13,14]$. In RR-MADD, the missense mutations lead to a misfolding of protein leading to decreased protein stability [15-17]. Riboflavin is the substrate that is converted to FAD. Increasing the availability of riboflavin, is thus speculated to promote folding and/or stabilizes the native conformation of certain types of ETF:QO variant proteins.

Here, we report a 65 year old male individual with diabetes presenting severe muscle weakness and exercise intolerance as major symptoms. Whole exome sequencing (WES) revealed that he is a compound heterozygous for two variants in the ETFDH gene, establishing the final diagnosis and responded to riboflavin supplementation. The study also identified a previously unreported variant in ACOT11 with highly-predicted functional impact.

\section{Case presentation}

The proband was a 65 year old patient with a relapsing and remitting course of lipid storage myopathy. His symptoms started at 17 years old when he was enlisted into the military. He noted muscle fatigue and exercise intolerance affecting only his lower limbs after intense exercise. These symptoms recurred with greater severity at 36 years old. His upper and lower limbs, as well as his neck muscles were affected. $\mathrm{He}$ also experienced difficulty in swallowing. Possible aggravating factors were irregular meals and heavy alcohol consumption. He was evaluated in another centre at that time and we were able to access part of his results. Creatinine kinase levels were elevated 20 times. Muscle biopsy histopathology showed the presence of fat globules within vacuolated muscle fibres and increased oxidative enzyme activates, suggesting a 'lipid storage disease'. He had received oral steroids intermittently, but no specific treatment was given as his symptoms resolved spontaneously. Recently, his symptoms recurred, and he was referred to our centre. In the past 2 years, he had picked up jogging and begun to experience similar episodes of muscle fatigue affecting only his lower limbs after exercise. Exercise intolerance had deteriorated and he can only manage $100 \mathrm{~m}$ of walking each time by the time he consulted our centre. No other muscles were affected. There were no accompanying cardiac or respiratory symptoms such as chest pain or breathlessness. His other medical problems included type 2 diabetes mellitus and hyperlipidemia. We noted he has a strong family history of type 2 diabetes mellitus, with 3 out of 5 siblings being inflicted with the disease. His elder son has multiple medical problems associated with obesity hypertension, aortic aneurysm, obstructive sleep apnea and stroke. His younger son has bicuspid aortic valve. There is no family history of consanguineous marriage or muscle disorders. To the best of our knowledge, his siblings and children do not suffer from any of the symptoms experienced by the proband (Fig. 1). Specifically, his siblings (RD-WES-002, RD-WES-003, RD-WES-

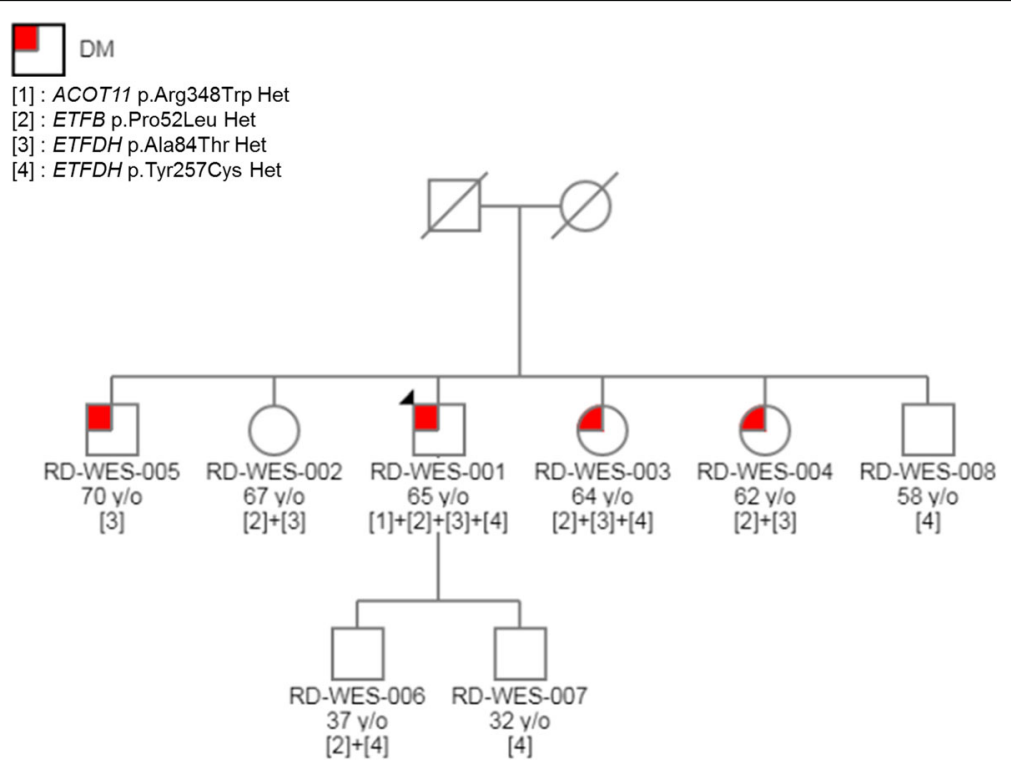

Fig. 1 Pedigree showing co-segregation of the heterozygous changes within ETFDH, ETFB and ACOT11 in the family. Open symbol represents unaffected subject. Patient (proband) is indicated with a black arrow 
004, RD-WES-005, and RD-WES-008) were determined as healthy based on their clinical phenotypes and normal creatinine kinase values.

On examination, he weighed $86.6 \mathrm{~kg}$ and his body mass index was $28.2 \mathrm{~kg} / \mathrm{m}^{2}$. There were no dysmorphic features. Cushingoid features were absent. Facial muscle weakness and ptosis were absent. Neck muscles were normal. There was no winging of both scapulae. Wasting of both quadriceps were noted. Muscle strength was reduced at the shoulders and the hips with manual muscle testing 4 out of 5 for both flexors and extensors. Distal muscle strength was normal for both upper and lower limbs (Table 1). Fatigability of the muscles was not demonstrated in the clinic. Muscle tone and reflexes were normal. He displayed normal gait. Examination of the cardiorespiratory and abdominal systems were normal.

Basic investigations revealed elevated values of creatinine kinase and myoglobin. Liver function tests, renal function tests and thyroid function tests were normal (Table 2). Tests specific to lipid storage disease that were performed included plasma acylcarnitines, serum carnitine level, serum riboflavin, urine acylglycines and urine organic acids. The results from the plasma acylcarnitines were abnormal, showing elevated concentrations of several acylcarnitine species (C5-C18) compared to reference range (Table 3).

\section{Clinical assessment and blood collection}

Clinical diagnosis was based on physical examination, muscle biopsy, biochemical tests. Peripheral blood samples were taken from the patient and his family members, including his 5 siblings and 2 children, aged 32 to 70. The study was according to the Declaration of Helsinki Principles and the ethical guidelines of our institution. Written informed consent was obtained prior to sample collection.

\section{Genetic testing}

Genomic DNA was extracted from peripheral blood using QIAamp DNA kit (Qiagen). Whole exome library preparation was performed for patient (RD-WES-001) and two siblings (RD-WES-005 and RD-WES-008) using Nimblegen SeqCap EZ Library SR kit (Roche) according to manufacturer instruction and enriched samples underwent paired-end sequencing on Illumina HiSeq 4000 device (Macrogen, Korea). Candidate variants were validated and followed up in the proband and his
Table 2 Laboratory results of the patient

\begin{tabular}{|c|c|c|c|}
\hline Tests & Results & Reference index & Units \\
\hline Albumin & 38 & $35-48$ & $g / L$ \\
\hline Alanine transferase & 37 & $17-63$ & $U / L$ \\
\hline Alkaline phosphatase & 46 & $38-126$ & $\mathrm{U} / \mathrm{L}$ \\
\hline Bilirubin & 12 & $7-31$ & umol/L \\
\hline Calcium & 2.28 & $2.15-2.58$ & $\mathrm{mmol} / \mathrm{L}$ \\
\hline \multicolumn{4}{|l|}{ Carnitine, serum } \\
\hline Carnitine, total & 47 & $34-78$ & $\mathrm{nmol} / \mathrm{ml}$ \\
\hline Carnitine, free (FC) & 28 & $25-54$ & $\mathrm{nmol} / \mathrm{ml}$ \\
\hline Acylcarnitine (AC) & 19 & $5-30$ & $\mathrm{nmol} / \mathrm{ml}$ \\
\hline AC/FC Ratio & 0.7 & $0.1-0.8$ & \\
\hline Creatinine & 66 & 60-105 & umol/L \\
\hline Creatinine kinase & 492 & $50-250$ & $U / L$ \\
\hline Glucose, fasting & 6.0 & $3.0-6.0$ & $\mathrm{mmol} / \mathrm{L}$ \\
\hline Glycated Hemoglobin & 6.4 & - & $\%$ \\
\hline \multicolumn{4}{|l|}{ Lipid Panel } \\
\hline Cholesterol & 5.2 & & $\mathrm{mmol} / \mathrm{L}$ \\
\hline $\mathrm{HDL}-\mathrm{C}$ & 1.4 & & $\mathrm{mmol} / \mathrm{L}$ \\
\hline LDL-C & 3.4 & & $\mathrm{mmol} / \mathrm{L}$ \\
\hline Triglycerides & 0.9 & & $\mathrm{mmol} / \mathrm{L}$ \\
\hline Myoglobin & 294 & $16-96$ & ug/L \\
\hline Riboflavin, serum & 6 & $1-19$ & $\mathrm{ug} / \mathrm{L}$ \\
\hline Thyroxine, free & 13 & $8-21$ & $\mathrm{pmol} / \mathrm{L}$ \\
\hline TSH & 2.69 & $0.34-5.60$ & $\mathrm{mIU} / \mathrm{L}$ \\
\hline $25-\mathrm{OH}$ vitamin D & 24 & $>20$ & $u g / L$ \\
\hline
\end{tabular}

Numbers out of reference index are in bold

relatives with Sanger-based sequencing. Primers used were shown in Additional file 1: Table S1.

The mapping of sequencing reads to NCBI GRCh37 human reference genome and variant detection were performed using the Burrows-Wheeler read aligner (BWA) and Genome Analysis Tool Kit (GATK), respectively. The detected single nucleotide polymorphisms (SNPs), deletion and insertion variants in coding regions and splice sites sequences were filtered by quality/depth, minimum total read depth (coverage). Variants passing these quality filters were annotated with information from public annotation databases using ANNOVAR. We exclude common variants with allele frequency greater than 0.05 in the 1000 Genomes and ExAC Browser. Choice of variants for further analysis were limited to genes implicated in

Table 1 Manual muscle testing (MMT) scale of upper limbs and lower limbs of the proband on examination

\begin{tabular}{|c|c|c|c|c|c|c|c|c|c|c|c|c|c|}
\hline & \multicolumn{2}{|l|}{ Neck } & \multicolumn{2}{|l|}{ Shoulders } & \multicolumn{2}{|l|}{ Elbows } & \multirow{2}{*}{$\begin{array}{l}\text { Fingers } \\
\text { Finger } \\
\text { grip }\end{array}$} & \multicolumn{2}{|l|}{ Hips } & \multicolumn{2}{|l|}{ Knees } & \multicolumn{2}{|l|}{ Ankles } \\
\hline & Flexion & $\overline{\text { Extension }}$ & Abduction & Adduction & Flexion & $\overline{\text { Extension }}$ & & Flexion & $\overline{\text { Extension }}$ & Flexion & $\overline{\text { Extension }}$ & Flexion & $\overline{\text { Extension }}$ \\
\hline $\begin{array}{l}\text { Power } \\
\text { (out of 5) }\end{array}$ & 5 & 5 & 4 & 5 & 5 & 5 & 5 & 4 & 4 & 5 & 5 & 5 & 5 \\
\hline
\end{tabular}


Table 3 Acylcarnitine profile of the patient before and after riboflavin treatment

\begin{tabular}{|c|c|c|c|c|}
\hline \multirow[t]{2}{*}{ Analytes } & \multicolumn{2}{|l|}{ Results } & \multirow{2}{*}{$\begin{array}{l}\text { Reference } \\
\text { range }\end{array}$} & \multirow[t]{2}{*}{ Units } \\
\hline & Before & After & & \\
\hline Co- Free carnitine & 36 & 42 & $17-64$ & umol/L \\
\hline C2- Acetylcarnitine & 10 & 3 & $2-17$ & umol/L \\
\hline C3-Propionylcarnitine & 0.42 & 0.31 & $0.14-0.95$ & umol/L \\
\hline C3DC-Malonylcarnitine & 0.03 & 0.03 & $0.01-0.08$ & umol/L \\
\hline C4-n-butyryl-/isobutyrylcarnitine & 0.33 & 0.16 & $0.08-0.46$ & umol/L \\
\hline $\mathrm{C} 4 \mathrm{OH}-3$-Hydroxy-Butyrylcarnitine & 0.12 & 0.06 & $0.01-0.24$ & umol/L \\
\hline C5-Isovaleryl-/2-Methylbutyrylcarnitine & 0.39 & 0.10 & $0.03-0.32$ & $\mathrm{umol} / \mathrm{L}$ \\
\hline C5:1-Tiglylcarnitine & 0.02 & 0.01 & $0.01-0.04$ & umol/L \\
\hline $\mathrm{C} 5 \mathrm{OH}-3-\mathrm{Hydroxy}$-Isovaerylcarnitine & 0.03 & 0.03 & $0.01-0.09$ & umol/L \\
\hline C5DC-Glutaryl/3-Hydroxydecanoylcarnitine & 0.28 & 0.06 & 0.010 .08 & umol/L \\
\hline C6-Hexanoylcarnitine & 0.43 & 0.25 & $0.02-0.12$ & umol/L \\
\hline C8-Octanoylcarnitine & 1.50 & 1.69 & $0.03-0.22$ & umol/L \\
\hline C10-Decanoylcarnitine & 3.07 & 1.82 & $0.05-0.42$ & umol/L \\
\hline C10:1-Decenoylcarnitine & 0.40 & 0.61 & $0.03-0.26$ & umol/L \\
\hline C10:2-Decadienoylcarnitine & 0.05 & 0.05 & $0.01-0.05$ & umol/L \\
\hline C12-Dodecanoylcarnitine & 0.87 & 0.15 & $0.02-0.13$ & umol/L \\
\hline C12:1-Dodecenoylcarnitine & 0.13 & 0.09 & $0.01-0.10$ & umol/L \\
\hline C14-Tetradecanoylcarnitine & 0.51 & 0.05 & $0.01-0.07$ & umol/L \\
\hline C14:1-Tetradecenoylcarnitine & 0.72 & 0.15 & $0.01-0.17$ & umol/L \\
\hline C14:2-Tetradecadienoylcarnitine & 0.20 & 0.07 & $0.01-0.05$ & umol/L \\
\hline C16-Hexadecanoylcarnitine & 0.81 & 0.13 & $0.06-0.24$ & umol/L \\
\hline C16:1-Hexadecenoylcarnitine & 0.53 & 0.05 & $0.01-0.07$ & umol/L \\
\hline C18-Octadecanoylcarnitine & 0.34 & 0.04 & $0.02-0.10$ & umol/L \\
\hline C18:1-Octadecenoylcarnitine & 0.95 & 0.12 & $0.05-0.28$ & umol/L \\
\hline C18:2-Linoleylcarnitine & 0.33 & 0.05 & $0.02-0.10$ & umol/L \\
\hline 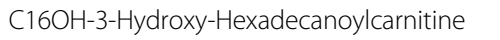 & 0.02 & 0.01 & $0.00-0.02$ & umol/L \\
\hline
\end{tabular}

Numbers out of reference range are in bold

glutaric academia, carnitine metabolism and acyl coenzyme metabolism; and supported by in silico analysis by different bioinformatics tools (PolyPhen2 and SIFT).

\section{Molecular findings}

The sequencing generated $67,408,294$ reads in the proband, 65, 560,602 in his older brother (RD-WES-005), and 18, 657, 150 in his younger brother (RD-WES-008). More than $99.9 \%$ of these reads were mapped and more than $74.7 \%$ were on-targets reads. Percentage of targets with more than 10X coverage in RD-WES-001, RD-WES-005 and RD-WES-008 were 95.5, 95.3 and 62. $4 \%$ respectively; and their corresponding mean depth were 57.9X, 56.0X and 16.4X. The number of variants identified per sample ranged from 81,341 to 105,643 (Additional file 1: Table S2). There was a total of 29,793 variants common across all 3 samples which may represent the genotypes shared among all the family members. These common variants were excluded from subsequent analysis, rendering a total of 3504 variants unique to the proband, 5986 variants common between proband and RD-WES-005, and 1500 variants common between proband and RD-WES-008. Although the specific mutations causing the myopathy syndrome are more likely to be found in set of patient specific mutations, there is a chance that causative mutations belong to the common variants shared with his siblings, if the patient is the only family member with the respective homozygous genotype. Among these, data annotation predicted 515 sample specific missense mutations in the proband, 314 missense variants common between proband and RD-WES-005, and 247 missense variants common between proband and RD-WES-008. No frameshift, insertion or deletion mutations were found. We further filtered these variants based on targeted pathways, minor allele frequency $(\mathrm{MAF} \leq 0.05$ in 1000 
Genome and ExAC databases) and predicted as damaging by two different computer algorithms, SIFT and PolyPhen2. As a result, we obtained a final list of 3 variants in the coding region of ETFDH and ACOT11 genes (Table 4). Among these, ETFDH c.250G > A (Ala84Thr) was characterized in the ClinVar database, as pathogenic. ETFDH c.770A > G (Tyr257Cys) was not reported in 1000 Genome or ExAC databases but has been reported with ETFDH c.250G > A in riboflavin-responsive lipid storage myopathy [8]. Hence, ETFDH Ala84Thr and Tyr257Cys represent excellent candidates as causative event underlying the trait. ACOT11 c.1042C $>\mathrm{T}$ (Arg348Trp) represents a missense variant that has not been reported in the literature in individuals with a LSM related disease. In silico analyses predict that this variant is probably damaging to protein structure and function.

All available members in the pedigree were screened for these three mutations. Sanger sequencing analysis validated the mutations in the proband (Fig. 2) and revealed that ACOT11 c. $1042 \mathrm{C}>\mathrm{T}$ variant was unique to him. In addition, one of his healthy sister (RD-WES003) harboured the same combination of ETFDH mutations. The other family members had only one of the heterozygous ETFDH mutations (data not shown).

\section{Structural analysis}

The 3-dimensional structure of the human ETF:QO protein was predicted using the SWISS-MODEL protein structure homology modelling server to evaluate the possible consequences of the missense mutation [18]. The porcine ETF:QO (PDB entry: $2 \mathrm{GMH}$ ) shared a high amino acid sequence similarity with the human homolog and was as the model template [13]. The program DeepView (Swiss Pdb viewer) was used for visualization and analysis of the modelled protein structure.

Structural analysis of ETF:QO suggests that the wildtype Ala84 located within the FAD binding domain and the replacement with threonine disrupted the stability of FAD binding, which is essential for the activation of ETF: QO [19]. To explore the impact of Ala84Thr and Tyr257Cys on protein function, human ETF:QO model was constructed using the published porcine ETF:QO crystal structure (PDB Id:2GMH) as the template (Fig. 3). Both Ala84 and Tyr257 are hydrophobic residues located in helices within the FAD binding domain. The newly introduced mutant residues differ in size and hydrophobicity values from the wild-type residues. These differences are predicted to disturb the protein conformation and hence binding properties of the FAD binding domain.

\section{Treatment}

The patient was given riboflavin $100 \mathrm{mg}$ thrice daily with significant improvement in symptoms. He also received dietary counseling for a low fat diet with advice against prolonged fasting. Clinical improvement was supported by normalization of serum creatinine kinase and myoglobin levels, as well as improvement in plasma long chain acyl carnitine results (Table 3).

\section{Discussion and conclusions}

In this report, we describe WES analysis of a patient with clinical features correspond to lipid storage myopathy and his healthy family members. Two compound heterozygous mutations in ETFDH gene were identified as the causative mutations. Within the ETFDH gene, the known pathogenic c.250G > A mutation was observed in homozygous and compound heterozygous state in several patients with MADD [8-10]. This variant (rs121964954) is more prevalent in Asians and reported in ExAC database with an allele frequency of $0.17 \%$ in East Asians. It has been reported in late-onset MADD patients from Asian countries including Taiwan, Hong Kong, Japan, Thailand and southern China [10-12, 20, $21]$. To date, this mutation has never been reported in Western countries [9]. The other variant ETFDH c. $770 \mathrm{~A}>\mathrm{G}$ was not present in ExAC database but reported in Chinese patients with MADD [22]. The study reported that the c.250G $>\mathrm{A}$ was more prevalent in Southern China, whereas c.770A > G had a higher frequency in Mainland China. Of note, ETFDH c.250G > A and c.770A $>\mathrm{G}$ mutations were also present in his healthy sister (RD-WES-003) who was 64 year old. Hence, RD-WES-003 may develop the symptoms associated with MADD in the future although the likelihoods are not high given her advance age. It is also possible that the compound heterozygous mutations in ETFDH can be tolerated or other genetic factors are required to cause disease.

Consistent with previous reports, supplementation of riboflavin, the precursor of FAD has strikingly improved the symptoms in this patient $[4,8,23,24]$. FAD is a

Table 4 Number of candidate variants

\begin{tabular}{|c|c|c|c|c|c|c|c|c|c|c|c|c|}
\hline \multirow[t]{2}{*}{ Gene } & \multirow[t]{2}{*}{ SNP ID } & \multirow{2}{*}{$\begin{array}{l}\text { Base } \\
\text { change }\end{array}$} & \multicolumn{5}{|c|}{1000 Genome } & \multirow[t]{2}{*}{ ExAC } & \multirow{2}{*}{$\begin{array}{l}\text { Protein } \\
\text { change }\end{array}$} & \multirow[t]{2}{*}{ SIFT } & \multirow[t]{2}{*}{ POLYPhen } & \multirow[t]{2}{*}{ Class } \\
\hline & & & AFR & AMR & EAS & EUR & SAS & & & & & \\
\hline ETFDH & rs121964954 & $250 G>A$ & 0 & 0 & 0.001 & 0 & 0 & 0.00173 & Ala84Thr & Deleterious & Damaging & Pathogenic \\
\hline ETFDH & - & $770 A>G$ & - & - & - & - & - & - & Tyr257Cys & Deleterious & Damaging & VUS \\
\hline ACOT11 & rs139751558 & $1042 C>T$ & 0 & 0 & 0.0198 & 0 & 0 & 0.04853 & Arg384Trp & Deleterious & Damaging & VUS \\
\hline
\end{tabular}




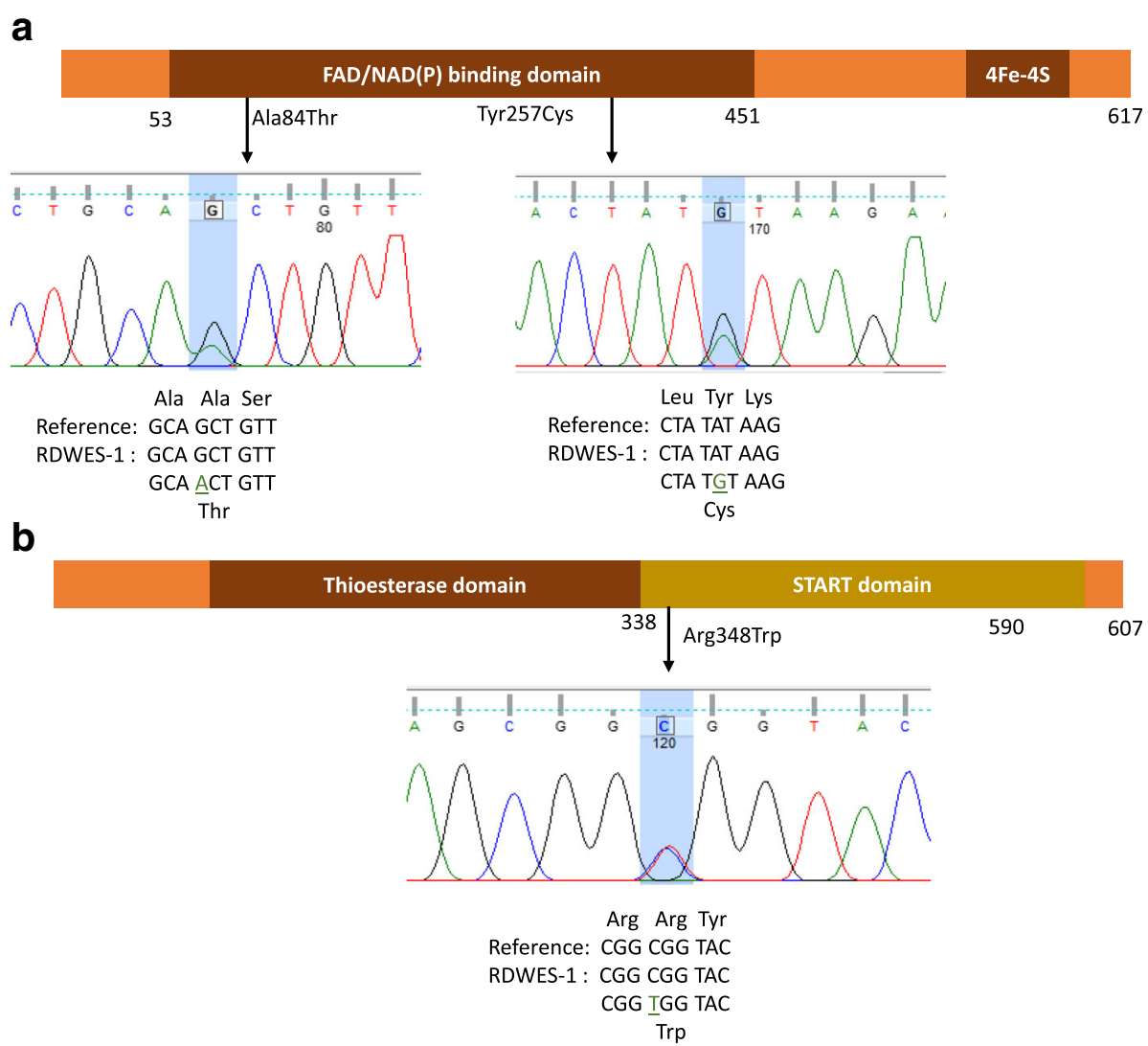

Fig. 2 Electropherograms and locations of missense mutations in ETFDH (a) and ACOT11 (b) sequences. Protein domains are schematized with numbers indicating the amino acid

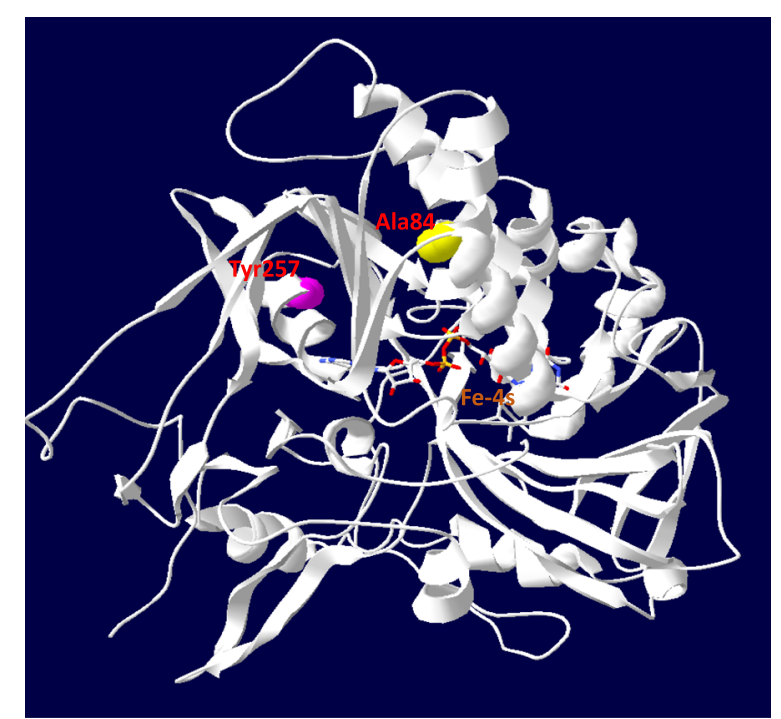

Fig. 3 Structure prediction of ETF:QO. The predicted wild-type model of human ETF:QO with Ala84 and Tyr257 in the hydrophobic FAD-binding domain shown in ball-and-stick representations cofactor for ETF:QO which is important for the enzyme catalytic activity, correct folding, assembly and protein stability [25]. The ETF:QO Ala84Thr and Tyr257Cys mutations are located in the FAD binding domain. According to the predicted ETF:QO 3D structure, these residues are buried within the core of the protein. Alteration at these positions represent replacement of hydrophobic residues (alanine and tyrosine) with polar residues (threonine and cysteine). These drastic changes are likely to alter the conformation proximate to the FAD-binding site and disrupt the stability of FAD binding, which is essential for enzyme activation. Although the mechanism of riboflavin efficacy in MADD patients is not yet completely clear, a likely explanation is that of riboflavin increases the intra-mitochondrial FAD concentration and enhances the conformational stabilization of the mutant ETF:QO protein. In turn, this could ameliorate the effect of the mutations that reduce the affinity of ETF:QO for FAD $[9,26]$.

ACOT11 has been implicated in regulating the oxidation of fatty acids [27]. The ACOT11 c.1042C > T variant (rs139751558) is ethnic-specific according to ExAC database, with an allele frequency of $4.85 \%$ in East Asians. The Arg348Trp variant resides in the START domain, which is 
involved in lipid binding and is essential for optimum enzyme activity [28]. The substitution represents a drastic change from a hydrophilic and positively charged residue to a hydrophobic and uncharged residue, which might impact lipid binding and impair oxidation of fatty acids. Intriguingly, this is the only patient specific variant that is predicted to be deleterious. However, without additional functional and/or genetic data, the significance of the alteration for disease is uncertain.

Due to its wide variety of clinical symptoms, LSM is difficult to diagnose. For cases highly suggestive of MADD, genetic analysis of ETFDH gene is highly recommended as vast majority of patients carry mutations in this gene [29]. For LSM, a large number of genes are implicated and targeted screening using Sanger sequencing will be an expensive and tedious option. In this study, we employed WES for comprehensive genetic diagnosis and have successfully revealed the mutations in ETFDH gene as the causal variants, leading to the personalized therapeutic strategy of riboflavin supplementation.

\section{Additional file}

Additional file 1: Table S1. Primers sequences used to amplify and sequence candidate genes. Table S2. Number of candidate variants filtered against dbSNP and 1000 Genome public databases. (DOCX $45 \mathrm{~kb}$ )

\section{Abbreviations}

BWA: Burrows-Wheeler read aligner; ETF: Electron transfer flavoprotein; ETF:QO: ETF:ubiquinone oxidoreductase; FAD: Flavin adenine dinucleotide; GATK: Genome Analysis Tool Kit; LSM: Lipid storage myopathy; MADD: Multiple acyl-CoA dehydrogenase deficiency; PCD: Primary carnitine deficiency; RR-MADD: Riboflavin-responsive MADD; WES: Whole-exome sequencing

\section{Acknowledgements}

We would like to thank the patients and their families for cooperation. We gratefully acknowledge Chet Hong Loh for performing the sequencing experiments.

\section{Funding}

This work was supported by the funding support from Singapore National Medical Research Council (NMRC/CG/017/2013).

\section{Availability of data and materials}

The datasets used and/or analysed during the current study are available from the corresponding author on reasonable request.

\section{Authors' contributions \\ $\mathrm{YL}$ and $\mathrm{RD}$ acquired and provided clinical data and samples from patient and his family. CWL constructed the family pedigree. EST analysed the data. SCL performed plasma acylcarnitine profiling. LLG designed the study, analysed most of the data and wrote the first draft of the manuscript. All authors have read, revised and approved the final manuscript.}

\section{Ethics approval and consent to participate}

Ethics approval was not applicable as test performed was used for clinical care. Written informed consent was obtained prior to sample collection.

\section{Consent for publication}

Written informed consent for publication of their clinical details was obtained from the patient.

\section{Competing interests}

The authors declare that they have no competing interests.

\section{Publisher's Note}

Springer Nature remains neutral with regard to jurisdictional claims in published maps and institutional affiliations.

\section{Author details}

${ }^{1}$ Molecular Diagnostic Laboratory, Tan Tock Seng Hospital, 11 Jalan Tan Tock Seng, Singapore 308433, Singapore. ${ }^{2}$ Department of Endocrinology, Tan Tock Seng Hospital, 11 Jalan Tan Tock Seng, Singapore 308433, Singapore.

${ }^{3}$ Department of Paediatrics, Genetics Services, KK Women's and Children's Hospital, 100 Bukit Timah Road, Singapore 229899, Singapore. ${ }^{4}$ Biochemical Genetics and National Expanded Newborn Screening, Department of Pathology and Laboratory Medicine, KK Women's and Children's Hospital, 100 Bukit Timah Road, Singapore 229899, Singapore. ${ }^{5}$ Lee Kong Chian School of Medicine, Nanyang Technological University, 11 Mandalay Road, Singapore 308232, Singapore. ${ }^{6}$ Yong Loo Lin School of Medicine, National University of Singapore, 12 Science Drive 2, Singapore 117549, Singapore.

Received: 4 October 2017 Accepted: 26 March 2018

Published online: 03 April 2018

\section{References}

1. Bruno C, Dimauro S. Lipid storage myopathies. Curr Opin Neurol. 2008; 21(5):601-6.

2. Frerman FE, Goodman SI. Defects of electron transfer flavoprotein and electron transfer flavoprotein-ubiquinone oxidoreductase: glutaric aciduria type II. In: Scriver CR, Beaudet AL, Sly WS, Valle D, Childs B, Kinzler KW, Vogelstein B, editors. In the metabolic and molecular bases of inherited disease. 8th ed. New York: McGraw-Hill; 2001. p. 2357-65.

3. Olsen RK, Andresen BS, Christensen E, Bross P, Skovby F, Gregersen N. Clear relationship between ETF/ETFDH genotype and phenotype in patients with multiple acyl-CoA dehydrogenation deficiency. Hum Mutat. 2003;22(1):12-23.

4. Grunert SC. Clinical and genetical heterogeneity of late-onset multiple acylcoenzyme a dehydrogenase deficiency. Orphanet J Rare Dis. 2014;9:117.

5. Goodman SI, Binard RJ, Woontner MR, Frerman FE. Glutaric acidemia type II: gene structure and mutations of the electron transfer flavoprotein:ubiquinone oxidoreductase (ETF:QO) gene. Mol Genet Metab. 2002;77(1-2):86-90.

6. Schiff M, Froissart R, Olsen RK, Acquaviva C, Vianey-Saban C. Electron transfer flavoprotein deficiency: functional and molecular aspects. Mol Genet Metab. 2006;88(2):153-8.

7. Yotsumoto Y, Hasegawa Y, Fukuda S, Kobayashi H, Endo M, Fukao T, Yamaguchi S. Clinical and molecular investigations of Japanese cases of glutaric acidemia type 2. Mol Genet Metab. 2008;94(1):61-7.

8. Wen B, Dai T, Li W, Zhao Y, Liu S, Zhang C, Li H, Wu J, Li D, Yan C. Riboflavin-responsive lipid-storage myopathy caused by ETFDH gene mutations. J Neurol Neurosurg Psychiatry. 2010;81(2):231-6.

9. Olsen RK, Olpin SE, Andresen BS, Miedzybrodzka ZH, Pourfarzam M, Merinero B, Frerman FE, Beresford MW, Dean JC, Cornelius N, et al. ETFDH mutations as a major cause of riboflavin-responsive multiple acyl-CoA dehydrogenation deficiency. Brain. 2007;130(Pt 8):2045-54.

10. Wang ZQ, Chen XJ, Murong SX, Wang N, Wu ZY. Molecular analysis of 51 unrelated pedigrees with late-onset multiple acyl-CoA dehydrogenation deficiency (MADD) in southern China confirmed the most common ETFDH mutation and high carrier frequency of c.250G >a. J Mol Med. 2011;89(6):569-76.

11. Lan MY, Fu MH, Liu YF, Huang CC, Chang YY, Liu JS, Peng CH, Chen SS. High frequency of ETFDH c.250G>a mutation in Taiwanese patients with late-onset lipid storage myopathy. Clin Genet. 2010;78(6):565-9.

12. Law LK, Tang NL, Hui J, Fung SL, Ruiter J, Wanders RJ, Fok TF, Lam CW. Novel mutations in ETFDH gene in Chinese patients with riboflavinresponsive multiple acyl-CoA dehydrogenase deficiency. Clin Chim Acta. 2009;404(2):95-9.

13. Zhang J, Frerman FE, Kim JJ. Structure of electron transfer flavoproteinubiquinone oxidoreductase and electron transfer to the mitochondrial ubiquinone pool. Proc Natl Acad Sci U S A. 2006;103(44):16212-7.

14. Simkovic M, Degala GD, Eaton SS, Frerman FE. Expression of human electron transfer flavoprotein-ubiquinone oxidoreductase from a baculovirus vector: kinetic and spectral characterization of the human protein. Biochem J. 2002;364(Pt 3):659-67.

15. Gregersen N, Bross P, Vang S, Christensen JH. Protein misfolding and human disease. Annu Rev Genomics Hum Genet. 2006;7:103-24.

16. Sato K, Nishina $Y$, Shiga K. In vitro refolding and unfolding of subunits of electron-transferring flavoprotein: characterization of the folding 
intermediates and the effects of FAD and AMP on the folding reaction. J Biochem. 1996;120(2):276-85.

17. Henriques BJ, Olsen RK, Bross P, Gomes CM. Emerging roles for riboflavin in functional rescue of mitochondrial beta-oxidation flavoenzymes. Curr Med Chem. 2010;17(32):3842-54.

18. Biasini M, Bienert S, Waterhouse A, Arnold K, Studer G, Schmidt T, Kiefer F, Gallo Cassarino T, Bertoni M, Bordoli L, et al. SWISS-MODEL: modelling protein tertiary and quaternary structure using evolutionary information. Nucleic Acids Res. 2014;42(Web Server issue):W252-8.

19. Er TK, Chen CC, Liu YY, Chang HC, Chien YH, Chang JG, Hwang JK, Jong YJ. Computational analysis of a novel mutation in ETFDH gene highlights its long-range effects on the FAD-binding motif. BMC Struct Biol. 2011;11:43.

20. Liang WC, Ohkuma A, Hayashi YK, Lopez LC, Hirano M, Nonaka I, Noguchi S, Chen LH, Jong YJ, Nishino I. ETFDH mutations, CoQ10 levels, and respiratory chain activities in patients with riboflavin-responsive multiple acyl-CoA dehydrogenase deficiency. Neuromuscul Disord. 2009;19(3):212-6.

21. Wasant $P$, Kuptanon C, Vattanavicharn N, Liammongkolkul S, Ratanarak P, Sangruchi T, Yamaguchi S. Glutaric aciduria type 2, late onset type in Thai siblings with myopathy. Pediatr Neurol. 2010;43(4):279-82.

22. Zhu M, Zhu X, Qi X, Weijiang D, Yu Y, Wan H, Hong D. Riboflavin-responsive multiple acyl-CoA dehydrogenation deficiency in 13 cases, and a literature review in mainland Chinese patients. J Hum Genet. 2014;59(5):256-61.

23. Vergani L, Barile M, Angelini C, Burlina AB, Nijtmans L, Freda MP, Brizio C, Zerbetto E, Dabbeni-Sala F. Riboflavin therapy. Biochemical heterogeneity in two adult lipid storage myopathies. Brain. 1999;122(Pt 12):2401-11.

24. Er TK, Liang WC, Chang JG, Jong YJ. High resolution melting analysis facilitates mutation screening of ETFDH gene: applications in riboflavinresponsive multiple acyl-CoA dehydrogenase deficiency. Clin Chim Acta. 2010;411(9-10):690-9.

25. Nagao M, Tanaka K. FAD-dependent regulation of transcription, translation, post-translational processing, and post-processing stability of various mitochondrial acyl-CoA dehydrogenases and of electron transfer flavoprotein and the site of holoenzyme formation. J Biol Chem. 1992; 267(25):17925-32.

26. Henriques BJ, Rodrigues JV, Olsen RK, Bross P, Gomes CM. Role of flavinylation in a mild variant of multiple acyl-CoA dehydrogenation deficiency: a molecular rationale for the effects of riboflavin supplementation. J Biol Chem. 2009;284(7):4222-9.

27. Tillander V, Alexson SEH, Cohen DE. Deactivating fatty acids: acyl-CoA Thioesterase-mediated control of lipid metabolism. Trends Endocrinol Metab. 2017;28(7):473-84.

28. Cohen DE. New players on the metabolic stage: how do you like them Acots? Adipocyte. 2013;2(1):3-6.

29. Angelini C, Tavian D, Missaglia S. Heterogeneous phenotypes in lipid storage myopathy due to ETFDH gene mutations. JIMD Reports. 2017;38: 33-40.

\section{Submit your next manuscript to BioMed Central and we will help you at every step:}

- We accept pre-submission inquiries

- Our selector tool helps you to find the most relevant journal

- We provide round the clock customer support

- Convenient online submission

- Thorough peer review

- Inclusion in PubMed and all major indexing services

- Maximum visibility for your research

Submit your manuscript at www.biomedcentral.com/submit

) Biomed Central 